\title{
Theoretical and Experimental Determination of the Maximum Scattering Cross-Section
} of Passive Linear Arrays

\author{
Appel-Hansen, Jørgen; Solymar, L.
}

Published in:

3rd European Microwave Conference

Link to article, DOI:

10.1109/EUMA.1973.331797

Publication date:

1973

Document Version

Publisher's PDF, also known as Version of record

Link back to DTU Orbit

Citation (APA):

Appel-Hansen, J., \& Solymar, L. (1973). Theoretical and Experimental Determination of the Maximum Scattering Cross-Section of Passive Linear Arrays. In 3rd European Microwave Conference (Vol. Volume 2, pp. 1-4). IEEE. https://doi.org/10.1109/EUMA.1973.331797

\section{General rights}

Copyright and moral rights for the publications made accessible in the public portal are retained by the authors and/or other copyright owners and it is a condition of accessing publications that users recognise and abide by the legal requirements associated with these rights.

- Users may download and print one copy of any publication from the public portal for the purpose of private study or research.

- You may not further distribute the material or use it for any profit-making activity or commercial gain

- You may freely distribute the URL identifying the publication in the public portal 
J. Appel-Hansen and L. Solymar

Laboratory of Electromagnetic Theory

Technical University of Denmark

DK-2800, Lyngby, Denmark

\section{Theory}

A linear array of $\mathrm{n}$ elements connected to a network of impedance matrix $\underline{z}_{m}$ is shown in Fig. 1. A plane wave incident from a direction $\theta_{1}$ is scattered by the array in a direction $\theta_{2}$ where $\theta_{1}$ and $\theta_{2}$ will be referred to as incident and scattering angles respectively. The amount of scattering between these two arbitrary directions is characterized by the relative scattering cross-section Asr (the scattering cross-section divided by the scattering cross-section of a single short-circuited element) which has recently been derived in the form [1]

$$
A_{s r}=R_{l l}^{2}\left|\underline{f}\left(\theta_{2}\right) \underline{\underline{Y}} \underline{\underline{f}}\left(\theta_{1}\right)\right|^{2}
$$

where $R_{\|}$is the self-resistance of the individual elements and the self-reactance is assumed to be zero,

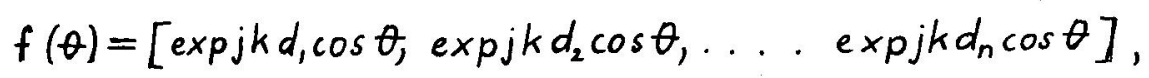

where $d_{1}, d_{2}, \ldots d_{n}$ are the distances of the elements from an arbitrary chosen zero, $\mathrm{k}=2 \pi / \lambda$, and $\lambda$ is the wavelength. The admittance matrix $\underline{y}$ connects the currents flowing in the elements with the induced voltages. It can be expressed with the aid of the mutual impedance matrix of the antenna $\underline{Z}_{a}$ and the impedance matrix of the network, $\underline{\underline{Z}}_{m}$ as follows

$$
\underline{\underline{Y}}=\left(\underline{\underline{Z}}_{a}+\underline{\underline{Z}}_{m}\right)^{-1}
$$

If we aim at maximum scattering it seems logical to avoid dissipation and choose reactive elements so that $\underline{\underline{Z}}_{m}=j \underline{\underline{x}}_{m}$.

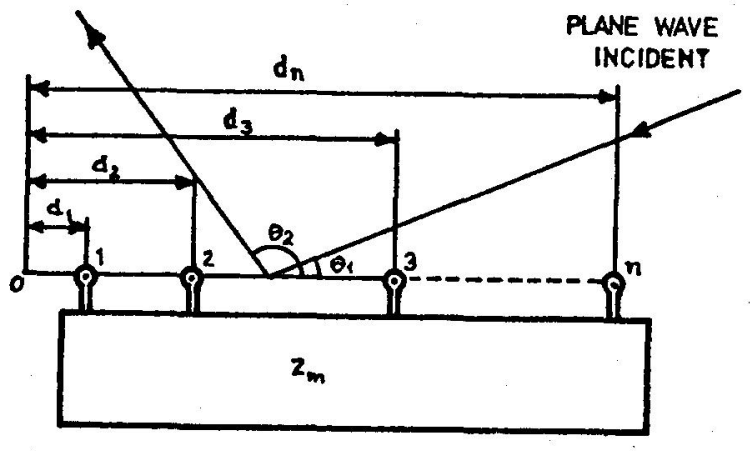

Fig. 1. Linear array Assuming that the geometry of the array and the incident and scattering angles are given, $\underline{f}\left(\theta_{1}\right), \underline{f}\left(\theta_{2}\right), R_{\|}$and $\underline{z}_{a}$ are fixed and the only variable at our disposal is the matrix $\underline{x}_{m}$ or more correctly all its elements (since $\underline{\underline{x}}_{m}$ is a symmetric matrix there are only $n(n+1) / 2$ independent elements for a 2 -port network). Unfortunately, there appears to be no general method with which the optimum values of $\left(\mathrm{X}_{m}\right)_{i k}$ and the resulting $A_{s r}\left(\theta_{1}, \theta_{2}\right)_{\text {max }}$ could be determined. The only analytical result we managed to

The authors wish to thank Ole Nielsen for making the computer calculations. Thanks are also due to $P$. Laugesen, $K$. Leschley and Fl. Hansen who performed most of the measurement work. 
prove for $n$ elements is for the case of specular scattering $\left(\theta_{2}=\pi-\theta_{1}\right)$; then the maximum relative scattering cross-section is obtained when all the mutual reactances are tuned out giving the value

$$
A_{s r}\left(\theta_{1}, \pi-\theta_{1}\right)_{\max }=G_{\max }^{2}\left(\theta_{1}\right)
$$

where $G_{\max }\left(\theta_{1}\right)$ is the maximum gain the array can produce in the direction $\theta_{1}$.

For two, three and four-element arrays we have used computer maximisation to get $A_{s r}\left(\theta_{1}, \theta_{2}\right)_{\max }$. Two examples are shown in Figs. 2 and 3 for the case when the arrays consist of two half-wave dipoles at distances $d=0.7 \lambda$ and $d=0.25 \lambda$. The variables are $\theta_{1}$, and $\theta_{2}$ and contour curves of $A_{s r}\left(\theta_{1}, \theta_{2}\right)_{\max }=$ const. are plotted (since the curves are symmetrical to the $\theta_{2}=\theta_{1}$ and $\theta_{2}=\pi-\theta_{1}$ diagonals, it is sufficient to plot one-quarter of the complete diagram). It may be seen that for $\mathrm{d}=0.7 \lambda$ the maximum occurs in the broadside direction $\left(\theta_{1}=\theta_{2}=90^{\circ}\right)$ whereas for $a=0.25 \lambda$ the direction of maximum scattering is in the forward direction $\left(\theta_{1}=180^{\circ}, \theta_{2}=0^{\circ}\right)$.

For $\mathrm{n}=2$ and for back-scattering $\left(\theta_{1}=\theta_{2}\right)$ an analytical form may be found

$$
A_{s r}\left(\theta_{1}, \theta_{1}\right)_{\max }=\frac{\cos ^{4} v}{\left(1+R_{a 12} / R_{11}\right)^{2}} \text { or } \frac{\sin ^{4} v}{\left(1-R_{a 12} / R_{11}\right)^{2}}
$$

whichever is the larger and $v=(k d / 2) \cos \theta_{1}$. The corresponding reactances are

$$
\left(X_{m}\right)_{11}=-\left(X_{m}\right)_{22}=-\left(R_{11}+R_{a / 2}\right) \tan v, \quad\left(X_{m}\right)_{11}=-\left(X_{m}\right)_{22}=\left(R_{11}-R_{a / 2}\right) \cot v \text {, }
$$

respectively, with the mutual reactances tuned out. It should be noted, however, that there are usually several networks capable to realise the maximum scattering cross-section. It is possible, for example, to obtain the maximum scattering cross-section for the broadside case with a network consisting of a transmission line of variable length connecting the two elements (Fig. 4a). For maximum backscattering from a direction $\theta_{1} \neq 90^{\circ}$, the more general network of Fig. $4 \mathrm{~b}$ must be used.

\section{Experiments}

The measurements were carried out at $3.3 \mathrm{GHz}$ in the Radio Anechoic Chamber of The Technical University of Denmark using the so-called overriding technique described in Reference [2]. A typical experimental set-up for determining the backscattering from two dipoles in the endfire case $\left(\theta_{1}=\theta_{2}=0\right)$ is shown in Fig. 5. As may be seen the network takes the form of Fig. $4 \mathrm{~b}$ capable to realise a general, loss-free, four-terminal network. In some of the experiments we used this general network although the majority of measured results was obtained with the stubtuner omitted.

In all experiments we could show the presence of disturbing reflections from the support and from the rather bulky network. The reflections from the network were reduced by arranging the network so as to give a minimum contribution to the measured back-scattering cross-section. In the particular example shown in Fig. 5 the linestretchers and the stubtuner were screened by absorbers.

In the broadside case $\left(\theta_{1}=\theta_{2}=90^{\circ}\right)$ the transmission lines were placed normal to the electric field vector of the incident wave. Moreover, in this case the linestretchers and the stubtuner were placed parallel to the direction of incidence.

The back-scattering cross-section for two elements in the broadside direction was measured for the inter-element spacings $d=0.49 \lambda$ and $d=0.71 \lambda$ as a function of the length of the interconnecting transmission line (Fig. 4a). The measured results are compared with the theoretical ones in Figs. 6 and 7 . For $d=0.7 / \lambda$ the maximum value of the back-scattering cross-section was further investigated by using the network of Fig. 4b. By varying the parameters of this general net- 


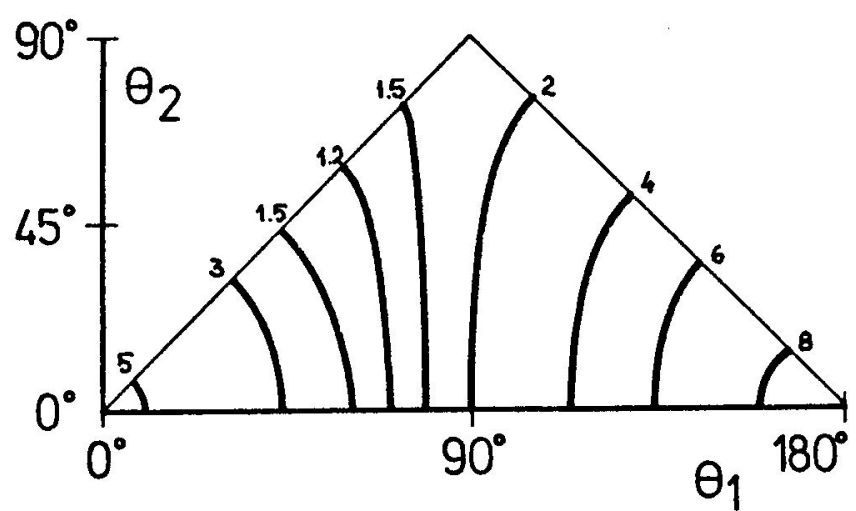

Fig. 2. Contour plot $d=0.25 \lambda$

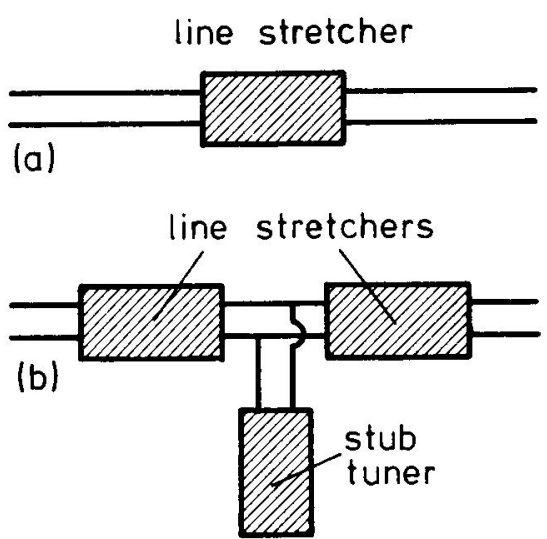

Fig. 4, a and b.

Connecting network

work the back-scattering cross-section was optimised. The value of this maximum was about $1 \mathrm{~dB}$ lower than the value $10.4 \mathrm{~dB} / \lambda^{2}$ obtained in Fig. 7 by using the simple network.

The results obtained for two dipoles in the endfire case $(d=0.25 \lambda)$ are shown in Fig. 8. In contrast to the broadside case the experimental values are now consistently below the theoretical ones.

For an array consisting of three elements we tried only the simple experiments in which all three dipoles were terminated in short-circuited transmission lines. For a distance of $0.8 \lambda$ between the elements the maximum occurred in the immediate vicinity of line lenghts giving a short-circuit at the terminals of the dipoles. The experimentally found value of the back-scattering cross-section was $13.3 \mathrm{~dB} / \lambda^{2}$ against the theoretical value of $13.1 \mathrm{~dB} / \lambda^{2}$

It may be seen from Figs. 6 - 8 that the experimental and theoretical curves vary in the same manner. The discrepancies are mainly due to scattering from 


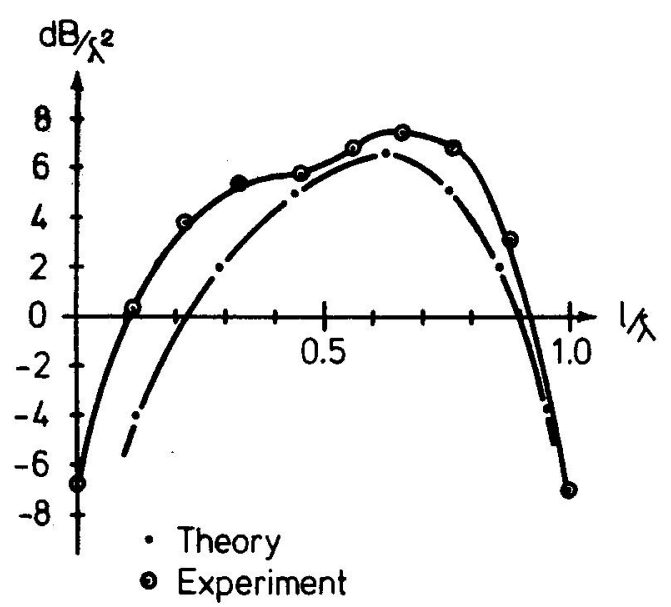

Fig. 6. Broadside, $d=0.49 \lambda$

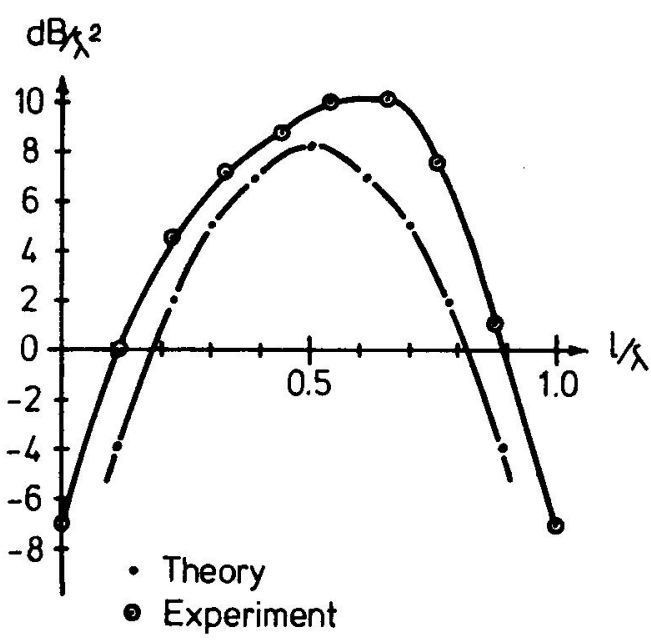

Fig. 7. Broadside, $d=0.71 \lambda$

the supporting structure and the connecting circuit but in addition there is, of course, a difference between the ideal half-wave array assumed in the theory and the one realised in practice. Finally we have to note that general measurement errors and inaccuracies in determining a reference level by means of reference spheres give one more possibility of explaining the discrepancies between theory and experiment.

\section{References}

[1] L. Solymar, On the scattering crosssection of passive linear arrays, Trans. IEEE PGAP-21, pp. 391-392, May 1973.

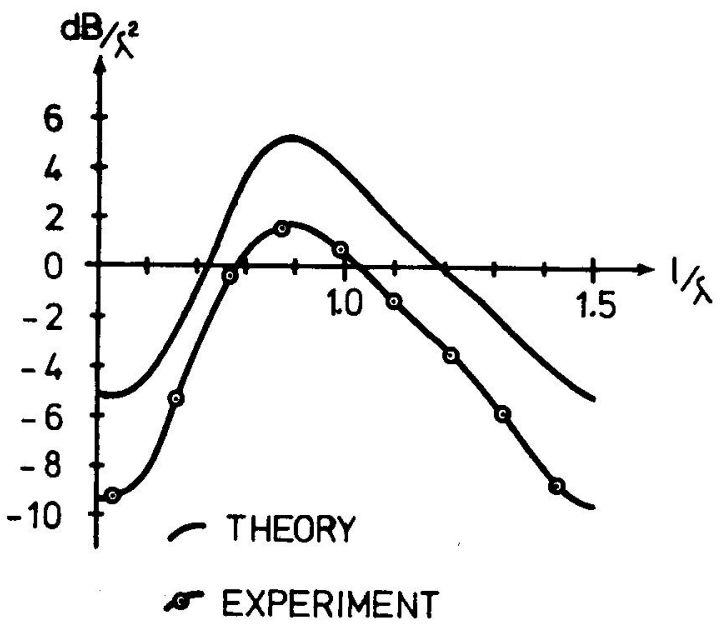

Fig. 8. Endfire, $d=0.25 \lambda$

[2] J. Appel-Hansen, A Van Atta reflector consisting of half-wave dipoles, Trans. IEEE PGAP-14, pp. 694-700, Nov. 1966. 